\title{
Aboriginal women, alcohol and the road to fetal alcohol spectrum disorder
}

\section{"The girls don't drink much; 'bout the same as the fellas"}

am an Aboriginal woman, with traditional connections to the Bidjara people from central western Queensland and extensive experience in working with Aboriginal women who consume alcohol during pregnancy.

During several decades of working in the health field, I have asked myself and others why Aboriginal people drink alcohol at such dangerous levels.

As I have reported earlier, ${ }^{1}$ I believe that the historical and political background and the cultural aspects of drinking have been insufficiently considered. There is an entrenched expectation of Aboriginal community members that to drink is an expression of identity and culture.

It is unrealistic to expect that individuals can take responsibility for their own actions outside the context of their cultural environment. Programs aimed at changing individual risky behaviour fail to acknowledge the way in which the person is inextricably tied to the culture in which he or she exists.

In many communities, alcohol use is a familiar and embedded practice that spans generations as well as individual lifetimes, from before birth to death. Its consequences are difficult to escape, whether a given person actually drinks or not.

Some years ago, with input from a number of Aboriginal community members, I constructed a framework to assist in understanding the development of identity and the resulting changes of emotions and physical boundaries across the lifespan. ${ }^{2}$

Using this framework, I have proposed an expanded view on the use of alcohol in Indigenous communities, ${ }^{2}$ formulated through interviews and discussions with members of remote, rural and urban Aboriginal communities in Queensland. This was originally done in the context of trying to better understand fetal alcohol spectrum disorder and early life trauma.

In this essay I use a narrative format to display dialogue, because objectivity of the "interviewer" and distance from the respondents' responses is not consistent with Aboriginal ways of knowing. Understanding the intersubjectivity between the author as an Aboriginal woman and the people whose input contributed to this report made possible the interactions and insights that follow. The impressions and conclusions should not be rejected on the grounds that the approach varied from more Westernised sociological research methods.

For many Aboriginal women, alcohol, like pregnancy, is a normal part of the life cycle.

During my research, young women and young men spoke honestly about the perceived relationship between alcohol and pregnancy, alcohol and drugs, and alcohol and crime, violence and abuse - all of which they associated

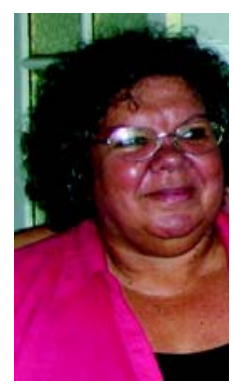

Lorian G Hayes

MAppEpid,

BAppHIthSc(Hons),

PhD Student

Centre for Chronic Disease, School of Medicine University of Queensland, Brisbane, QLD.

l.hayes3@uq.edu.au

doi: 10.5694/mjall.10390 with their families, relationships, friends and daily environment.

Within this life cycle the relationship between alcohol and pregnancy was revealed to be more complex than the physical effects of either. Young people strongly confirmed their connection with their toxic social environment and were aware of the hardships and disadvantages that confront them daily. The issues they identified were family breakdown; community disharmony; family and community dysfunction; alcohol and drug addiction; teenage pregnancy; peer pressure; violence within the home and community; unemployment; shame, pain and anger within; a sense of isolation and not belonging; lack of trust and respect from family, friends and peers; the high incidence of rape and sexual abuse; and the lack of opportunities to gain education and training within the community.

My proposed life cycle framework or model broadens the Western approach and integrates with cultural constructionist theories to give a clearer understanding of alcohol use. ${ }^{2}$

The following story-lines provide examples of how interview respondents conceptualise their own or others' health status in terms of historical, cultural and systemic impediments.

\section{Stamping the story on the kids (age 0-2 years)}

The early years will shape the life's journey for many children who were not only exposed to alcohol in utero, but were also born into an environment that was awash with alcohol and violence.

If basic human needs are met through nurturing and responsible care, children will develop hopefulness, cheerfulness, trust, confidence and security. If children do not have caring experiences, they will develop insecurity, a feeling of worthlessness and general mistrust.

Although few individuals remember explicit stories from their lives before the age of 24 months, experiences within this period are generally accepted as having a significant impact on the development of emotional and psychological wellbeing.

\section{The disappearing childhood (age $3-5$ years)}

It is during the ages of $3-5$ years that children begin to retain strong memories that continue to provide background to their emotions in later life. Generally, children develop responsibility for self-sufficiency between the ages of 3 and 5. An important aspect of this is the development of trust. Trust in adults and their ability to ensure one's safety in a crisis is the earliest form of faith. If 
caregivers are inconsistent in satisfying a child's needs, the child will not develop this sense of trust, faith and hope. ${ }^{3}$

Children whose needs are not met feel that they have been abandoned by their carers, as depicted in the story below.

\section{Story 1. Small, alone and scared}

When one is a tiny little boy and is sent to bed by his mumma, who is drinking noisily in the next room where the music is loud, then suddenly, all becomes very quiet and still. You pull the blankets up over your head and lie very still because you become really scared. Too scared to move. You lay there thinking "Is the bogeyman's gunna come an get me?" You call out to your mumma and dadda but there is no answer. You suddenly realise that you are all alone in the house. Thoughts wander through your little mind, wondering where is mumma and dadda? Where are they? Why don't they hear my call? They must know I am scared? What should I do now? Should I stay here or should I try and run to find my nanna's or auntie's place? It is very dark outside. I awake to hear a very loud crash and yelling - people fighting. My uncle then comes in and carries me over to my nanna's house. Here I know that I am safe. There is no reason to be scared anymore. ${ }^{1}$

Interviewees reported feeling alone as a child, even when surrounded by adults drinking and partying, resulting in the child feeling unimportant as an individual. As these children grew older, they told of becoming more dependent on friends and peers for acceptance; their behaviour mimicking that of the adults around them and the peers for whose attention they aspired. Sometimes they reported an extraordinary sense of isolation related to what they perceived as a breakdown of their cultural identity, as well as the lack of mutual respect between older and younger community members, creating a sense of shame.

\section{The "walk-the-talk" stage (age 6-8 years)}

Between the ages of 6 and 8 years, the child develops a sense of responsibility for self-care and care of others. In crisis situations where the adult does not provide an environment of safety, a child may take on the responsibility of care for younger children.

\section{Story 2 . The protective older child}

As a child who lived in a home where there is lots of violence, bashings and too much grog, because I was the bigger kid I used to get all the smaller kids in a room and we would lock the door and go into the corner and huddle together, we would cover our ears and cry and I would rock to try and silence the noise and screams from my mum asking my dad to stop. I am now in my early twenties and I don't rock anymore. Loud noises still frighten us kids; we were so scared, so very, very scared. Why did our mum and dad do this to us? We had no one to come and take us away to somewhere safe; it was like nobody cared. ${ }^{2}$

In addition to the impact on the child, I have observed that when a child takes on a parental role for children younger than him- or herself, including siblings and

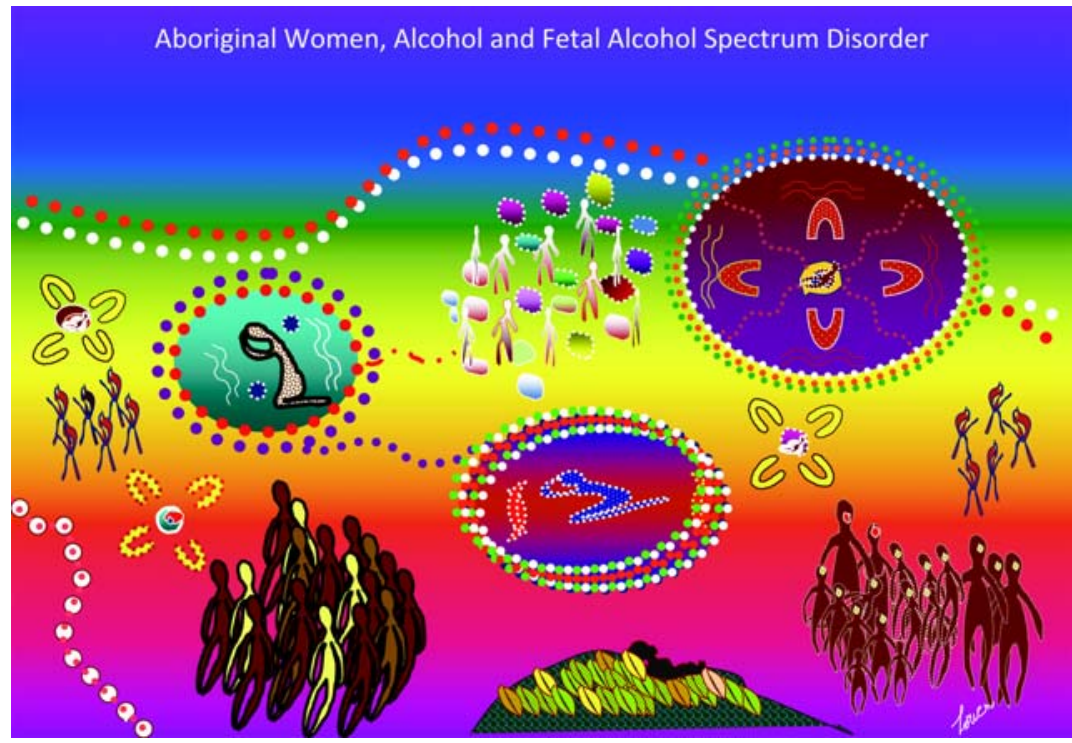

cousins, this relieves adults of their responsibilities and thus impairs the family's ability to provide nurturing and responsible care.

\section{Feelings of failure (age 8-11 years)}

During the mid school-age years (8-11 years), children learn about their successes and failures from interactions within their environment. This can be measured in how strong a power base they have built over the younger children and who they control with financial rewards of lollies, drinks and takeaway food. The following story depicts the sense of isolation and alienation this brings from a young age.

\section{Story 3. Shame, failure and alienation}

Jodie is four years old and has been outside playing with some older kids. Her sisters told her they had to hurry to school, but Jodie knows that they are going to the flats up the road. She wants to follow them and they scream back at her, "Get home you f... little so-and-so". Jodie stops in her tracks. She feels intense shame as she looks about to see if anyone has heard. A group of old men sit smoking in a front yard nearby and they show no sign of acknowledgement. Jodie knows they must have heard. She wanders back home to look for breakfast. As she enters the front door she can smell the stale grog from the night before. There is nothing in the kitchen for her to eat. She notices her mother lying quietly on the couch. Her mother mumbles something about a drink and Jodie climbs onto the kitchen bench to put the kettle on. She feels bad for her mother and worse for herself.

\section{"I'm clearin" out" (age 12-13 years)}

By the time children reach the youth stage (11-13 years), they begin to mimic the behaviour of adults around them. Then, as young teenagers, they perceive themselves to be (and may be accepted by the community as) true adults. 


\section{Story 4. An empty belly}

You go to bed quivering with fear and listening to drunks all night. You wake up and there are drunks everywhere, sleeping all around, and some still drinking. You search for food to fill your empty belly before you go to school. Most times there is none. Usually you go to school with an empty belly. You feel tired and you get a pain in your belly from lack of food. You become shy and embarrassed and begin to isolate yourself from others, especially those who have food. You run home at lunchtime hoping that there is some food waiting, but there is never any. When you come home from school, there are drunks still there. You go to bed and the drunks are still there — same old cycle. Eventually, after being exposed to alcohol year after year, you give up and join in, fill your empty belly with grog and become a drunk too. ${ }^{1}$

The above account depicts the emotions of a child whose basic needs, such as food, are not met. As the child grows older, he or she begins to realise that survival depends on achieving independence.

\section{The cycle continues (alcohol and pregnancy)}

The reasons why women continue to drink alcohol while pregnant are varied and complex. The pregnancy itself is a validation of adult status, as is the consumption of alcohol. At the same time, the pregnancy, often at a young age, is an additional determinant of social disadvantage.

\section{Story 5. A vicious cycle}

She may have been raped. Especially being young girls, they're trying to heal their own problems. So they look for the first person to come along, looking for good faces. They drink beers an' wine, it is cheaper. Except when they really want to party out and look for a man, they get spirits. They get pregnant and then they forget to stop drinking. They have money problems, which leads to drinking, which leads to pregnancy, which leads to pension, which leads to drinking, which leads to more problems, which leads to more drinking, which leads to more pregnancies - then they can't look after their kids. ${ }^{1}$

The strategies described in such stories reflect both dependence and independence. Young women feel isolation and a desire to be loved. A man of their own and a baby can provide adult status, along with money gained through either the relationship or through social security payments. If the relationship becomes violent and/or the man is unsupportive, the drinking resumes and the cycle continues. Under these circumstances, the baby's dependency becomes overwhelming and burdensome.

\section{Story 6. Violence causing harm}

I don't think they know if anything can happen to the baby. Something might happen when they drink if they are pregnant. Drinking alcohol is a way women try to kill their babies. Some young women get drunk and even try to commit suicide, not just because they are pregnant - there is other abuse too. The person drinks alcohol — becoming angry — and then picks a fight with another woman or a man and becomes involved in a fight — killing the baby one time. ${ }^{1}$

In this example, fetal death removes full responsibility from the mother. A community elder told me that removing fault is common, with the woman claiming she does not remember or that it was beyond her control, as she was drunk. Members of the community will in turn come together to support the young woman go through grief at the loss of her child.

Both young women and young men whom I interviewed felt that the practice of drinking alcohol when pregnant is seen as a way to kill the baby, either directly or indirectly (by initiating a cycle of violence in the hope that it will cause a miscarriage). They did not otherwise implicate or acknowledge toxic side effects of the alcohol consumed, and the concept of fetal alcohol syndrome or other fetal alcohol effects was not of concern to them.

A whole range of factors contribute to a cycle in which alcohol is accepted as an inevitable part of life and death. The community does not perceive a special problem with women's drinking, either in amount or in the drinking patterns, manifestations and toxic side effects. Drinking is the expected community norm.

The girls don't drink much; 'bout the same as the fellas (quote from a community member)

Being a drinker is not equivalent to being an Aboriginal. When Aboriginal people enter into a drinking cycle, even the unborn child is affected.

If the cycle of drinking in Aboriginal communities is to be broken, a more effective model for health promotion would be to aim towards acknowledging children who are continually exposed to examples of the negative adult behaviour associated with alcohol. It should be designed to enhance skills in developing positive patterns of behaviour for later life and negating the effects of witnessing irresponsible adult behaviour.

Provenance: Not commissioned; not externally peer reviewed. This essay was an entrant in the 2011 Dr Ross Ingram Memorial Essay Competition. It is adapted from an article published in the Aboriginal and Islander Health Worker Journal.' Quotes are reproduced with permission.

1 Hayes LG. Grog babies: where do they fit in this alcohol life cycle? Aboriginal Islander Health Worker J 2001; 25: 14-17.

2 Hayes LG. Children of the grog [unpublished honours thesis]. Brisbane: University of Queensland, 1998.

3 Erikson EH. Identity: youth and crisis. New York: Norton, 1968. 